\title{
Prognostic role of translocator protein and oxidative stress markers in chronic lymphocytic leukemia patients treated with bendamustine plus rituximab
}

\author{
ANIELLO DE ROSA ${ }^{1}$, SILVIA ZAPPAVIGNA ${ }^{2}$, MARIA ROSARIA VILLA ${ }^{3}$, SALVATORE IMPROTA $^{3}$, \\ ELENA CESARIO $^{2}$, LUCIA MASTRULLO ${ }^{3}$, MICHELE CARAGLIA ${ }^{2}$ and PAOLA STIUSO ${ }^{2}$ \\ ${ }^{1}$ Local Sanitary Agency, Naples 3 Southern, Torre del Grecco, Naples I-80059; ${ }^{2}$ Department of Biochemistry, \\ Biophysics and General Pathology, Second University of Naples, Naples I-80138; \\ ${ }^{3}$ Hematology Unit, San Gennaro Hospital, Naples I-80131, Italy
}

Received November 10, 2014; Accepted November 26, 2014

DOI: $10.3892 / \mathrm{ol} .2014 .2817$

\begin{abstract}
Principally located in the outer mitochondrial membrane, the translocator protein (TSPO) is an $18-\mathrm{kDa}$ transmembrane protein that is a key component of the mitochondrial permeability transition pore. TSPO is associated with a number of biological processes, including apoptosis, the regulation of cellular proliferation, porphyrin transport and heme biosynthesis, immunomodulation, anion transport and the regulation of steroidogenesis. Thus, numerous studies have proposed TSPO as a promising target for novel therapeutic agents, particularly for the treatment of cancer. In the present study, the response of 30 consecutive chronic lymphocytic leukemia (CLL) patients to bendamustine and rituximab treatment was evaluated according to TSPO expression levels. Furthermore, thiobarbituric acid reactive substances (TBARS) and nitric oxide (NO) levels, as well as caspase-3 activity were determined. Compared with the lymphocytes of healthy donors, the 30 consecutive CLL patients exhibited increased TSPO expression levels, decreased TBARS and NO levels and reduced caspase-3 activity. Six months after the treatment commenced, the TSPO/mitochondria ratio resembled that of the healthy controls in 24/30 CLL patients. In addition, an increase in TBARS and NO levels, two markers of oxidative
\end{abstract}

Correspondence to: Professor Michele Caraglia, Department of Biochemistry, Biophysics and General Pathology, Second University of Naples, Via L. De Crecchio 7, Naples I-80138, Italy

E-mail: michele.caraglia@unina2.it; michele.caraglia@alice.it

Abbreviations: CLL, chronic lymphocytic leukemia; CR, complete remission; MPTP, mitochondrial permeability transition pore; $\mathrm{NO}$, nitric oxide; NR, no remission; PR, partial remission; PBMC, peripheral blood mononuclear cells; TBARS, thiobarbituric acid reactive substances; TSPO, translocator protein; VDAC, voltage-dependent anion channel

Key words: apoptosis, chronic lymphocytic leukemia, prognostic factor, translocator protein, NO, TBARS stress, and a potentiation of caspase-3 activity in all responder patients was observed. Notably, the six patients who appeared to be resistant to treatment also displayed higher TSPO levels, and lower caspase-3 activity and TBARS levels. These data indicate that TSPO expression may be a molecular prognostic factor in CLL patients.

\section{Introduction}

Previously termed the peripheral benzodiazepine receptor, the translocator protein (TSPO) is a key element of the mitochondrial permeability transition pore (MPTP), which is a multiprotein complex located at the contact sites between the inner and outer mitochondrial membranes of various different cell types, including cells of the hematopoietic system. TSPO is physically associated with the backbone of MPTP via its adenosine nucleotide translocase and voltage-dependent anion channel (VDAC) (1). Consistent with its localization in the MPTP, TSPO has been implicated in various mithochondrial functions, such as the control of respiration, the modulation of inner membrane ion channel activities, as well as the regulation of apoptosis, cell proliferation and a number of other associated processes (2). It has previously been demonstrated that overexpression of TSPO inhibits apoptosis induced by free radicals or ultraviolet light. Concordantly, TSPO ligands have been demonstrated to induce caspase-3 (3) and -9 activation, the cytosolic release of cytochrome $c$ and cause an increase in the production of reactive oxygen species (ROS), resulting in the activation of p38 mitogen activated protein kinase and the opening of the MPTP (4). Furthermore, Carayon et al (5) demonstrated that TSPO expression correlated with $\mathrm{H}_{2} \mathrm{O}_{2}$ cytotoxicity resistance in hematopoietic cell lines (5); following the transfection of Jurkat cells with human TSPO complementary DNA, $\mathrm{H}_{2} \mathrm{O}_{2}$ resistance increased compared with wild-type cells. These results indicate that TSPO may aid in preventing free radical-induced cellular damage of mitochondria and may regulate cell apoptosis in the hematopoietic system.

Previously, TSPO and its ligands have been highlighted as potential targets for the development of novel anticancer agents (6). TSPO is highly expressed in various cancer cell 
types, including colon (7), brain (8), breast (9), ovary (10-11) and liver (12) cancer, and its expression is particularly high in organs involved in steroidogenesis (13). Although additional studies are required to improve the understanding of the biological functions of TSPO, clear evidence exists regarding its involvement in steroid biosynthesis. Furthermore, TSPO is key in cancer cell growth (14-17), with Maaser et al (18) demonstrating that specific TSPO ligands are able to induce apoptosis and cell cycle arrest in colorectal cancer cells.

Additionally, TSPO is regarded as a potential prognostic factor in cancer. In particular, it has been reported that lymphoma cell lines (19-20) and myeloid/lymphoid cells obtained from leukemia patients $(5,20)$ express high levels of TSPO, and ovarian, hepatic and colonic carcinomas, as well as glioma $(7-8,10-12)$ have demonstrated increased TSPO densities compared with the corresponding healthy tissue. In specific cases, TSPO expression correlates with the grade of the tumor malignancy and patient survival (21); for example, relatively high levels of TSPO density were observed in more rapidly proliferating breast cancer cells (22) and more aggressive breast cancer phenotypes (23).

CLL is characterized by the accumulation of mature malignant cluster of differentiation (CD) $5^{+}$B-lymphocytes Despite the use of numerous agents in the treatment of CLL, patients may develop mechanisms of resistance (24). In order to stratify patients according to their potential to respond to targeted therapy, personalized targeted therapy has been proposed; however, this requires the identification of novel (bio)markers.

In the present study, the role of TSPO as prognostic factor in CLL patients was investigated by evaluating the response to bendamustine and rituximab treatment according to TSPO expression. In addition, thiobarbituric acid reactive substance (TBARS) and nitric oxide (NO) levels, as well as caspase- 3 activity, were analyzed in the lymphocytes of healthy donors compared with CLL patients.

\section{Materials and methods}

Preclinical characteristics, treatment strategy and peripheral blood mononuclear cell (PBMC) isolation. The present study included 10 healthy blood bank donors and 30 patients from the Hematology Unit of San Gennaro Hospital (Naples, Italy). The patients enrolled in the present study were diagnosed with CLL according to the following criteria: Monotypic expansion of lymphoid cells $\left(\geq 15 \times 10^{3} / \mu 1\right)$, morphologically consistent with CLL (small lymphocytes), in the blood for $\geq 60$ days prior to treatment; $>30 \%$ lymphocytes in the bone marrow; and normal renal (creatinine, $<2.0 \mathrm{mg} / \mathrm{dl}$ ) and hepatic (bilirubin, $<2.0 \mathrm{mg} / \mathrm{dl}$ ) function. No patients had previously been treated for CLL and no patients had previously received four cycles of bendamustine plus rituximab. The treatment strategy administered in the present study was intravenous rituximab $\left(375 \mathrm{mg} / \mathrm{m}^{2}\right)$ every 28 days, followed by intravenous bendamustine $\left(90 \mathrm{mg} / \mathrm{m}^{2}\right)$ on the next day, for two consecutive days. This treatment regimen was continued until the occurrence of disease progression or unacceptable levels of toxicity.

The response criteria used was that previously defined by the National Cancer Institute Working Group (25). Complete remission (CR) was defined as the absence of all palpable disease and the return of the blood counts to within the following normal ranges: Neutrophils, $>1.5 \times 10^{3} / \mathrm{ml}$; platelets, $>100 \times 10^{3} / \mathrm{ml}$; hemoglobin, $>11 \mathrm{~g} / \mathrm{dl}$; and bone marrow aspirate lymphocyte percentage, $<30 \%$. Partial remission (PR) was defined as a 50\% decrease in palpable disease accompanied by a $50 \%$ improvement in all abnormal blood parameters. Patients were considered to be responsive to the treatment if a PR of $\geq 6$ months was observed and resistant to the treatment if a PR of $<6$ months or no remission (NR) were observed. PBMCs were isolated from blood samples obtained prior to (T0), one week after (T1) and six months after (T6) the commencement of treatment. The PBMCs were isolated using Ficoll density gradient centrifugation, as previously described (26) and stored at $-80^{\circ} \mathrm{C}$ for use in the following experiments. In addition, sera was collected from the patients at the abovementioned time periods and stored at $-20^{\circ} \mathrm{C}$ for use in the following experiments. This study was approved by the ethics committee of San Gennaro Hospital and written informed consent was obtained from all patients.

Analysis of TSPO protein expression levels by flow cytometry. The PBMCs were fixed for $20 \mathrm{~min}$ in a $3 \%(\mathrm{w} / \mathrm{v})$ paraformaldehyde (PFA) solution and permeabilized for 10 min with $0.1 \%$ (w/v) Triton X-100 in phosphate-buffered saline (PBS) at room temperature. To prevent non-specific interactions occurring between antibodies, the cells were treated with 5\% bovine albumin serum (BSA) in PBS for $2 \mathrm{~h}$ and incubated with a specific mouse monoclonal antibody raised against TSPO [cat. no. SAB1405525; dilution, 1:1,000 in blocking solution; 3\% (w/w) BSA in 0.1\% Tris-buffered saline-Tween; Sigma-Aldrich, St. Louis, MO, USA] for $2 \mathrm{~h}$ at $37^{\circ} \mathrm{C}$. Following multiple washes, the cells were incubated with a secondary IgG goat anti-mouse monoclonal antibody (cat. no. A-11001; Alexa Fluor ${ }^{\circledR} 488$; Life Technologies, Grand Island, NY, USA) diluted 1:1,000 in blocking solution for $1 \mathrm{~h}$ at room temperature. The samples were subsequently washed twice and, for each sample, 10,000 cells were counted using a FACSCalibur $^{\mathrm{TM}}$ flow cytometer (BD Biosciences, Franklin Lakes, NJ, USA) and CellQuest ${ }^{\mathrm{TM}}$ software (BD Biosciences). As TSPOs are expressed on mitochondria, the cellular mitochondrial content of TSPO was determined as follows: The cells were fixed with $3 \%$ PFA, labeled with $10^{-7} \mathrm{~mol} / 1$ nonyl acridine orange for $15 \mathrm{~min}$ at room temperature, washed twice in PBS and analyzed using flow cytometry. Thus, the mitochondrial TSPO density was determined by calculating the number of TSPO sites per unit of mitochondrial mass.

Nitrite assay. Nitric oxide (NO) is a molecular mediator of numerous physiological processes, including vasodilation, inflammation, thrombosis, immunity and neurotransmission. Thus, a number of methods exist, which analyze NO levels in biological systems. Under physiological conditions, $\mathrm{NO}$ is rapidly converted into the stable end products nitrite (NO2-) and nitrate and subsequently the hematic concentrations are frequently assessed as an index of systemic NO production. Therefore, nitrite levels were measured using the Griess reaction, as previously described by Gomez-Monterrey et al (27).

TBARS levels. The PBMC samples were incubated with $0.5 \mathrm{ml}$ of $20 \%$ acetic acid (pH 3.5) and $0.5 \mathrm{ml}$ of $0.78 \%$ aqueous 
thiobarbituric acid solution. The mixture was heated to $95^{\circ} \mathrm{C}$ for $45 \mathrm{~min}$ and centrifuged at 1,600 $\mathrm{x} \mathrm{g}$ for $5 \mathrm{~min}$. To quantify the amount of TBARS in the supernatant fractions, spectrophotometry was performed at an absorbance of $532 \mathrm{~nm}$ (27) and data were expressed as TBARS/serum protein in $\mu \mathrm{M} / \mu \mathrm{g}$. Data are presented as the average of triplicate measurements from duplicate experiments.

Caspase-3 activity. The BD ApoAlert ${ }^{\mathrm{TM}}$ Caspase-3 Fluorogenic assay kit (BD Biosciences Clontech, Palo Alto, CA, USA) and a fluorescent microplate reader (Applied Biosystems Life Technologies, Foster City, CA, USA) were used to determine the caspase-3 activity levels, as previously described (28).

Statistical analysis. Statistical analyses were conducted by performing an analysis of variance with Neumann-Keul's multiple comparison test or the Kolmogorov-Smirnov test, as appropriate. Analyses of the differences between the CR and NR patients were performed using the Mann-Whitney U test for non-parametric independent and continuous variables and all data are expressed as the mean \pm standard deviation. ${ }^{*} \mathrm{P}<0.01$ indicates a statistically significant difference between the control and CLL patients.

\section{Results}

Patient characteristics and clinical response to therapy. A total of 30 patients were enrolled from the Hematology Unit of San Gennaro Hospital. The pretreatment clinical characteristics of the 30 patients are indicated in Table I. Among the 30 enrolled patients, $67 \%$ were male and the median age was 73 years, with $48 \%$ patients aged $\sim 65$ years and $52 \%$ patients aged $\geq 75$ years. Compared with the healthy patients, the CLL patients exhibited a significantly higher median white blood cell count $\left(75,000 / \mathrm{mm}^{3} ; \mathrm{P}=0.004\right)$, a marginally lower median hemoglobin level $(11.5 \mathrm{~g} / \mathrm{dl} ; \mathrm{P}=0.05)$ and a marginally higher median platelet count $\left(180,000 / \mathrm{mm}^{3} ; \mathrm{P}=0.05\right)$.

The CLL patients received four cycles of treatment with bendamustine plus rituximab. Patients were considered to be responsive to the treatment if a $\mathrm{PR}$ of $\geq 6$ months was observed and resistant to the treatment if a PR of $<6$ months or NR were observed. Following six months of treatment with bendamustine plus rituximab, 12/30 (40\%) patients achieved CR, eight $(26 \%)$ patients achieved PR, six (20\%) patients achieved no remission (NR) and four (13\%) patients succumbed to the disease (Table II).

Modulation of TSPO expression. To evaluate the use of TSPO as a therapeutic target and prognostic marker, the response of CLL patients to bendamustine and rituximab treatment was determined according to TSPO expression. As TSPO is located on the mitochondria, the cellular mitochondrial content was determined by performing flow cytometry analysis; furthermore, TSPO density at the mitochondrial level was calculated by the number of TSPO sites per unit of mitochondrial mass.

TSPO expression was evaluated at T0 and T6. Compared with the lymphocytes of the healthy participants, the leukemic cells of the 30 CLL patients exhibited an increased level of TSPO, normalized for mitochondrial expression (Fig. 1A).
Table I. Pretreatment clinical characteristics $(n=30)$.

\begin{tabular}{lc}
\hline Variable & Value \\
\hline Median age, years & 73 \\
Age distribution, \% & \\
65 years & 48 \\
$75-80$ years & 50 \\
$\geq 80$ years & 2 \\
Gender distribution, $\%$ & \\
Male & 67 \\
Female & 33 \\
Median white blood cell count, $/ \mathrm{mm}^{3}$ & 78,000 \\
Median hemoglobin level, $\mathrm{g} / \mathrm{dl}$ & 11.5 \\
Median platelet count, $/ \mathrm{mm}^{3}$ & 180,000 \\
\hline
\end{tabular}

Table II. Clinical response to therapy $(n=30)$.

\begin{tabular}{lr} 
Clinical response & Patients, n (\%) \\
\hline Complete remission & $12(40)$ \\
Partial remission & $8(26)$ \\
No remission & $6(20)$ \\
Mortality & $4(13)$
\end{tabular}

Six months after the treatment commenced, a decrease in the TSPO/mitochondria ratio occurred, resembling that of the healthy controls in 24/30 CLL patients (Fig. 1A). CR patients exhibited low TSPO levels at T0, which were marginally reduced following six months of treatment compared with the NR group (Fig. 1B). Notably, the six patients who were resistant to treatment (NR patients) displayed significantly $(\mathrm{P}<0.01)$ higher TSPO levels compared with $\mathrm{CR}$ patients at T0; furthermore, the NR TSPO levels were significantly $(\mathrm{P}<0.01)$ reduced following six months of therapy (Fig. 1B). Thus, the results indicate an inverse trend in the TSPO levels between the two groups of patients.

Modulation of serum levels of NO, TBARS and caspase-3 activity. The present study evaluated TBARS and NO levels, two markers of oxidative stress, in the lymphocytes of $30 \mathrm{CLL}$ patients at T0, T1 and T6.

At T0, lower levels of NO (Fig. 2) and marginally higher levels of TBARS (Fig. 3) were identified in the CLL patients compared with the healthy controls. Bendamustine plus rituximab therapy at the T1 timepoint did not significantly change the serum NO levels (Fig. 2) but did slightly increase the TBARS level (Fig. 3); however, NO (Fig. 2) and TBARS (Fig. 3) mean serum levels were significantly $(\mathrm{P}<0.0001)$ increased in all responder patients $(24 / 30)$ at T6. TBARS but not NO levels exceeded the mean values recorded in healthy subjects. In addition, apoptosis was evaluated by detecting caspase-3 activity in the CLL patients. Prior to treatment (T0), low caspase-3 activity was identified in the CLL patients compared with the healthy donors; however, one week after treatment 
A

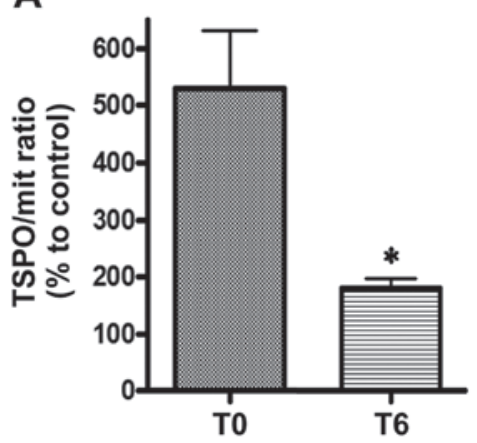

B

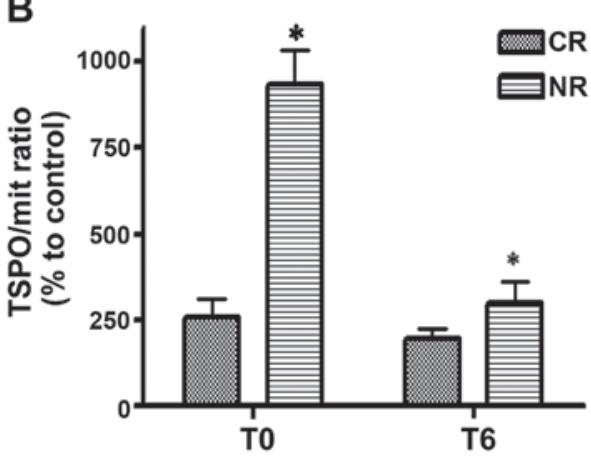

Figure 1. Evaluation of the variation in translocator protein (TSPO) expression in 30 patients with CLL. TSPO density at the mitochondrial level was obtained by calculating the number of TSPO sites per unit of mitochondrial mass. (A) Changes in TSPO expression in 30 CLL patients with respect to healthy patients at T0 and T6. (B) Changes in TSPO expression in CR and NR patients with respect to healthy patients at T0 and T6. Error bars represent the standard deviation of the mean. ${ }^{*} \mathrm{P}<0.01, \mathrm{NR}$ at T0 vs. CR at T0, NR at T0 vs. $\mathrm{CR}$ at T6, NR at T0 vs. NR at T6 and NR at T6 vs. CR at T6. CR, complete remission; NR, no remission; Mit, mitochondria; PBR, peripheral-type benzodiazepine receptor; T0, before treatment commenced; T6, six months after treatment commenced.

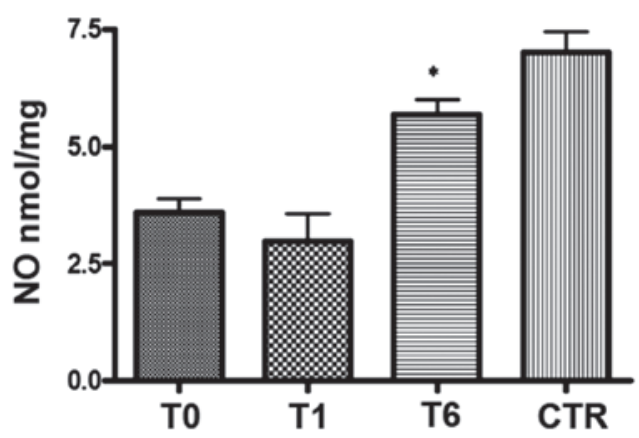

Figure 2. Serum NO protein expression level variation in 24 responder patients compared with healthy patients using an enzyme spectrophotometric assay. Error bars represent the standard deviation of the mean. ${ }^{*} \mathrm{P}<0.01 \mathrm{vs.} \mathrm{T0,}$ T1 and CTR. CTR, healthy patients; NO, nitric oxide; T0, before treatment; T1, one week after treatment commenced; T6, six months after treatment commenced.

commenced, an increase in caspase-3 activity was observed in all of the responder patients (24/30) and an additional increase was observed six months after the commencement of treatment (Fig. 4).

Of note, the six patients who appeared to be resistant to treatment displayed lower caspase-3 activity and TBARS

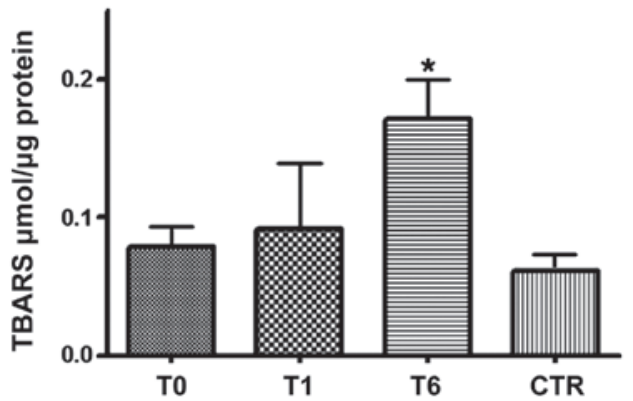

Figure 3. TBARS protein expression level variation in 24 responder patients compared with healthy patients using an enzyme spectrophotometric assay. Error bars represent the standard deviation of the mean. ${ }^{*} \mathrm{P}<0.01$ vs. T0, T1 and CTR. CTR, healthy patients; T0, before treatment; T1, one week after treatment commenced; T6, six months after treatment commenced; TBARS, thiobarbituric acid reactive substance.

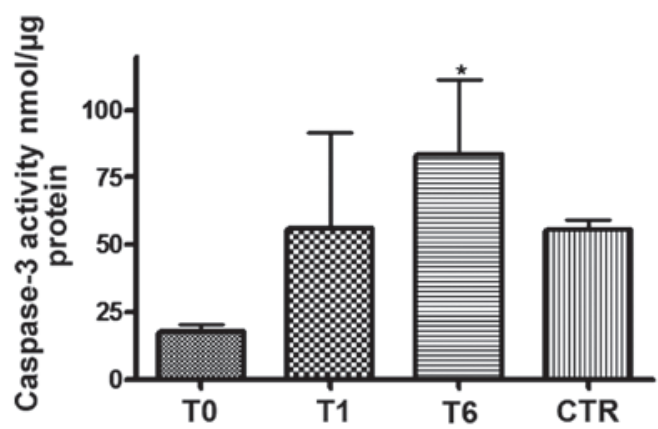

Figure 4. Caspase-3 activity variation in 24 responder patients compared with healthy patients using an enzyme spectrophotometric assay. Error bars represent standard deviation of the mean. ${ }^{*} \mathrm{P}<0.01$ vs. T0 and CTR. CTR, healthy patients; T0, before treatment; T1, one week after treatment commenced; T6, six months after treatment commenced.

levels six months after therapy (data not shown). These data indicate again that the increase in NO, TBARS and caspase-3 activity levels recorded following six months of treatment may be predictive of a response to therapy.

\section{Discussion}

TSPO is a component of the MPTP (with other protein constituents, such as VDAC) and is involved in a number of biological processes, including apoptosis, the regulation of cellular proliferation, porphyrin transport and heme biosynthesis, immunomodulation, anion transport, and the regulation of steroidogenesis. In addition, TSPO facilitates the regulation of the release of apoptotic factors into the cytosol; this correlation between TSPO, and the occurrence of programmed cell death and apoptotic onset was identified by studying the role of TSPO in the MPTP (29). TSPO causes prolonged opening of the MPTP and the release of apoptotic factors, such as Smac, cytochrome $c$ and apoptosis-inducing factor $(30,31)$, from the mitochondria into the cytosol, resulting in osmotic swelling of the mitochondrial matrix, and dysregulation of ATP synthesis and oxidative phosphorylation. Ultimately, apoptosis and necrotic signaling cascades are activated and cell death occurs (32), highlighting the correlation between TSPO and the protection of cells from 
apoptosis. Notably, previous studies have identified an association between TSPO and cancer; for example, it was demonstrated that TSPO overexpression induced by transfection protects lymphocytes against ultraviolet light-induced cell apoptosis (33), and TSPO expression was correlated with the ability of breast cancer cells to grow in severe combined immunodeficient mice (34). Furthermore, it was demonstrated that TSPO positively correlates with proliferation rate but inversely correlates with spontaneous apoptosis rates in various glioma cell lines. In patients exhibiting tumors with TSPO overexpression, TSPO may contribute to a poor prognosis by mediating proliferative and/or apoptosis-protective effects. However, these functions of TSPO may be reversed by the administration of TSPO-specific exogenous ligands in a variety of tumor types (14-18); furthermore, increased binding capacities of TSPO-specific ligands have been reported in colorectal cancer (18) and a variety of different tumor types (14-16). However, TSPO protein overexpression was only directly determined in astrocytoma and breast cancer. In addition, a study of 86 astrocytoma patients identified that TSPO protein expression correlated with the tumor grade (9) and, in a small number of breast cancer samples and cell lines, TSPO protein expression correlated with malignant cancer (21).

In the present study, TSPO expression levels were evaluated in 30 CLL patients treated with bendamustine plus rituximab. Following six months of treatment, 12/30 (40\%) patients achieved CR, eight $(26 \%)$ patients achieved PR, six $(20 \%)$ achieved no remission (NR) and four (13\%) succumbed to the disease. TSPO levels were higher in leukemic cells compared with the lymphocytes of healthy individuals at T0; however, six months after treatment commenced, the present study identified a decrease in the TSPO/mitochondria ratio in 24/30 CLL patients to resemble that of the healthy controls. In addition, CR patients exhibited low TSPO levels at T0 compared with NR patients. Thus, the results of the present study indicate an inverse trend in TSPO expression levels in the two groups of patients.

The role of NO varies in cancer biology and is dependent on a number of factors; NO may be involved in the promotion or prevention of tumor occurrence, depending on the time of exposure to NO, the tumor microenvironment and the concentration of NO. Low concentrations of NO (range, 1-30 nM) produced high levels of cyclic guanosine monophosphate, promoting tumor angiogenesis and the proliferation of endothelial cells, and a wider range of NO concentrations (range, 0-100 nM) corresponded to an increase in the activity of the proliferative and anti-apoptotic Akt and Erk-dependent pathways in tumor cells $(35,36)$, appearing to enhance angiogenesis and protect tumor cells from apoptosis. Thus, at NO concentrations of $0-100 \mathrm{nM}$, the molecules activated by $\mathrm{NO}$ are considered to be correlated with poor prognosis events in cancer. By contrast, higher NO levels (>300 $\mathrm{nM}$ ) appeared to promote apoptosis and anti-tumor activity.

In the present study, a spectrophotometric assay was performed to determine the serum NO levels of CLL patients treated with bendamustine plus rituximab. Six months after (T6) treatment commenced, responsive patients exhibited a strong increase in NO and TBARS levels, as well as a potentiation of caspase-3 activity. Notably, the six patients who were resistant to treatment exhibited lower caspase-3 activity and TBARS levels. These data again indicate that the increase in NO and TBARS levels, as well as caspase-3 activity levels, recorded at T6 may be predictive of a response to therapy. Notably, in responsive patients, the increased NO levels possibly had an anti-tumor and pro-apoptotic role, as demonstrated by the corresponding increase in caspase- 3 activity.

In conclusion, these data indicate that TSPO expression may be a molecular prognostic marker in CLL patients. In addition, TSPO may represent a useful therapeutic target for increasing treatment efficacy in CLL patients.

\section{References}

1. McEnery MW, Snowman AM, Trifiletti RR and Snyder SH: Isolation of the mitochondrial benzodiazepine receptor: association with the voltage-dependent anion channel and the adenine nucleotide carrier. Proc Natl Acad Sci 89: 3170-3174, 1992

2. Casellas P, Galiegue $\mathrm{S}$ and Basile AS: Peripheral benzodiazepine receptors and mitochondrial function. Neurochem Int 40: 475-486, 2002.

3. Sutter AP, Maaser K, Höpfner M, et al: Specific ligands of the peripheral benzodiazepine receptor induce apoptosis and cell cycle arrest in human esophageal cancer cells. Int J Cancer 102: 318-327, 2002.

4. Zisterer, Campiani G, Nacci V and Williams DC: Pyrrolo-1,5-benzoxazepines induce apoptosis in HL-60, Jurkat, and Hut-78 cells: a new class of apoptotic agents. J Pharmacol Exp Ther 293: 48-59, 2000.

5. Carayon P, Portier M, Dussossoy D, Bord A, Petitprêtre G, Canat X, Le Fur G and Casellas P: Involvement of peripheral benzodiazepine receptors in the protection of hematopoietic cells against oxygen radical damage. Blood 87: 3170-3178, 1996.

6. Austin CJ, Kahlert J, Kassiou M and Rendina LM: The translocator protein (TSPO): a novel target for cancer chemotherapy. Int J Biochem Cell Biol 45: 1212-1216, 2013.

7. Katz Y, Eitan A and Gavish M: Increase in peripheral benzodiazepine binding sites in colonic adenocarcinoma. Oncology 47: 139-142, 1990.

8. Cornu P, Benavides J, Scatton B, Hauw JJ and Philippon J: Increase in omega 3 (peripheral-type benzodiazepine) binding site densities in different types of human brain tumours. A quantitative autoradiography study. Acta Neurochir (Wien) 119: 146-152, 1992.

9. Hardwick M, Fertikh D, Culty M, Li H, Vidic B and Papadopoulos V: Peripheral-type benzodiazepine receptor (PBR) in human breast cancer: correlation of breast cancer cell aggressive phenotype with PBR expression, nuclear localization, and PBR-mediated cell proliferation and nuclear transport of cholesterol. Cancer Res 59: 831-842, 1999.

10. Katz Y, Ben-Baruch G, Kloog Y, Menczer J and Gavish M: Increased density of peripheral benzodiazepine-binding sites in ovarian carcinomas as compared with benign ovarian tumours and normal ovaries. Clin Sci (Lond) 78: 155-158, 1990.

11. Batra S and Iosif CS: Elevated concentrations of mitochondrial peripheral benzodiazepine receptors in ovarian tumors. Int J Oncol 12: 1295-1298, 1998.

12. Venturini I, Alho H, Podkletnova I, Corsi L, Rybnikova E, Pellicci R, Baraldi M, Pelto-Huikko M, Helén P and Zeneroli ML: Increased expression of peripheral benzodiazepine receptors and diazepam binding inhibitor in human tumors sited in the liver. Life Sci 65: 2223-2231, 1999.

13. Papadopoulos V: Peripheral-type benzodiazepine/diazepam binding inhibitor receptor: biological role in steroidogenic cell function. Endocr Rev 14: 222-240, 1993.

14. Wu X and Gallo KA: The 18-kDa translocator protein (TSPO) disrupts mammary epithelial morphogenesis and promotes breast cancer cell migration. PLoS One 8: e71258, 2013.

15. Beinlich A, Strohmeier R, Kaufmann M and Kuhl H: Relation of cell proliferation to expression of peripheral benzodiazepine receptors in human breast cancer cell lines. Biochem Pharmacol 60: 397-402, 2000. 
16. Landau M, Weizman A, Zoref-Shani E, Beery E, Wasseman L, Landau O, Gavish M, Brenner S and Nordenberg J: Antiproliferative and differentiating effects of benzodiazepine receptor ligands on B16 melanoma cells. Biochem Pharmacol 56: 1029-1034, 1998.

17. Ikezaki K and Black KL: Stimulation of cell growth and DNA synthesis by peripheral benzodiazepine. Cancer Lett 49: 115-120, 1990.

18. Maaser K, Höpfner M, Jansen A, et al: Specific ligands of the peripheral benzodiazepine receptor induce apoptosis and cell cycle arrest in human colorectal cancer cells. Br J Cancer 85: 1771-1780, 2001.

19. Alexander BE, Roller E and Klotz U: Characterization of peripheral-type benzodiazepine binding sites on human lymphocytes and lymphoma cell lines and their role in cell growth. Biochem Pharmacol 44: 269-274, 1992.

20. Canat X, Carayon P, Bouaboula M, Cahard D, Shire D, Roque C, Le Fur G and Casellas P: Distribution profile and properties of peripheral-type benzodiazepine receptors on human hemopoietic cells. Life Sci 52: 107-118, 1993.

21. O'Brien ER, Kersemans V, Tredwell M, Checa B, Serres S, Soto MS, Gouverneur V, Leppert D, Anthony DC and Sibson NR: Glial activation in the early stages of brain metastasis: TSPO as a diagnostic biomarker. J Nucl Med 55: 275-280, 2014.

22. Beinlich A, Strohmeier R, Kaufmann M and Kuhl H: Specific binding of benzodiazepines to human breast cancer cell lines. Life Sci 65: 2099-2108, 1999.

23. Hardwick M,FertikhD, Culty M,LiH, Vidic B and Papadopoulos V: Peripheral-type benzodiazepine receptor (PBR) in human breast cancer: correlation of breast cancer cell aggressive phenotype with PBR expression, nuclear localization, and PBR-mediated cell proliferation and nuclear transport of cholesterol. Cancer Res 59: 831-842, 1999.

24. Chiorazzi N, Rai KR and Ferrarini M: Chronic lymphocytic leukemia. N Engl J Med 352: 804-815, 2005.

25. Cheson BD, Bennett JM, Grever M, Kay N, Keating MJ, O'Brien S and Rai KR: National Cancer Institute-sponsored Working Group guidelines for chronic lymphocytic leukemia: revised guidelines for diagnosis and treatment. Blood 87: 4990-4997, 1996.

26. Correale P, Campoccia G, Tsang KY, et al: Recruitment of dendritic cells and enhanced antigen specific immune-reactivity in cancer patients treated with hr-GM-CSF (Molgramostim) and hr-IL-2: results from a phase Ib clinical trial. Eur J Cancer 37: 892-902, 2001.
27. Gomez-Monterrey I, Campiglia P, Scognamiglio I, Vanacore D, Dicitore A, Lombardi A, Caraglia M, Novellino E and Stiuso P: DTNQ-Pro, a mimetic dipeptide, sensitizes human colon cancer cells to 5-fluorouracil treatment. J Amino Acids 2013: 509056, 2013.

28. Tenore GC, Manfra M, Stiuso P, Coppola L, Russo M, Ritieni A and Campiglia P: Polyphenolic pattern and in vitro cardioprotective properties of typical red wines from vineyards cultivated in Scafati (Salerno, Italy). Food Chem 140: 803-809, 2013.

29. Veenman L and Gavish M: The role of $18 \mathrm{kDa}$ mitochondrial translocator protein (TSPO) in programmed cell death, and effects of steroids on TSPO expression. Curr Mol Med 12: 398-412, 2012

30. Veenman L, Papadopoulos V and Gavish M: Channel-like functions of the 18-kDa translocator protein (TSPO): regulation of apoptosis and steroidogenesis as part of the host-defense response. Curr Pharm Des 13: 2385-2405, 2007.

31. Parker MA, Bazan HE, Marcheselli V, Rodriguez de Turco EB and Bazan NG: Platelet-activating factor induces permeability transition and cytochrome c release in isolated brain mitochondria. J Neurosci Res 69: 39-50, 2002.

32. Papadopoulos V, Baraldi M, Guilarte TR, et al: Translocator protein $(18 \mathrm{kDa})$ : new nomenclature for the peripheral-type benzodiazepine receptor based on its structure and molecular function. Trends Pharmacol Sci 27: 402-409, 2006.

33. Stoebner P E, Carayon P, Casellas P, Portier M, Lavabre-Bertrand T, Cuq P, Cano JP, Meynadier J and Meunier L: Transient protection by peripheral benzodiazepine receptors during the early events of ultraviolet light-induced apoptosis. Cell Death Differ 8: 747-753, 2001.

34. Hardwick M, Rone J, Han Z, Haddad B and Papadopoulos V: Peripheral-type benzodiazepine receptor levels correlate with the ability of human breast cancer MDA-MB-231 cell line to grow in SCID mice. Int J Cancer 94: 322-327, 2001.

35. Ridnour LA, Thomas DD, Switzer C, et al: Molecular mechanisms for discrete nitric oxide levels in cancer. Nitric Oxide 19: 73-76, 2008

36. Prueitt RL, Boersma BJ, Howe TM, et al: Inflammation and IGF-I activate the Akt pathway in breast cancer. Int J Cancer 120: 796-805, 2007. 